\title{
MAXIMAL SUBGROUPS OF SPORADIC GROUPS
}

\author{
ROBERT A. WILSON
}

\begin{abstract}
A systematic study of maximal subgroups of the sporadic simple groups began in the 1960s. The work is now almost complete, only a few cases in the Monster remaining outstanding. We give a survey of results obtained, and methods used, over the past 50 years, for the classification of maximal subgroups of sporadic simple groups, and their automorphism groups.
\end{abstract}

\section{INTRODUCTION}

The subtitle of the 'Atlas of Finite Groups' 7 is 'Maximal Subgroups and Ordinary Characters for Simple Groups'. These two aspects of the study of finite simple groups remain at the forefront of research today. The Atlas was dedicated to collecting facts, not to providing proofs. It contains an extensive bibliography, but not citations at the point of use, making it difficult for the casual reader to track down proofs. In the ensuing 30 years, moreover, the landscape has changed dramatically, both with the appearance of new proofs in the literature, and with the ability of modern computer algebra systems to recompute much of the data in the twinkling of an eye.

As far as maximal subgroups are concerned, shortly before the publication of the Atlas it became clear that the maximal subgroup project should be extended to almost simple groups. The reason for this is that it is not possible to deduce the maximal subgroups of an almost simple group directly from the maximal subgroups of the corresponding simple group. This was made clear by the examples described in [49, especially perhaps the maximal subgroup $S_{5}$ of $M_{12}: 2$, which is neither the normalizer of a maximal subgroup of $M_{12}$, nor the normalizer of the intersection of two non-conjugate maximal subgroups of $M_{12}$.

The results on maximal subgroups for all the classical groups in the Atlas, as well as exceptional groups of types ${ }^{2} B_{2},{ }^{2} G_{2}, G_{2}$ and ${ }^{3} D_{4}$, are proved, and in many instances corrected, in the recent book by Bray, Holt and Roney-Dougal 3. This leaves the sporadic groups and five exceptional groups, ${ }^{2} F_{4}(2)^{\prime}, F_{4}(2)$, $E_{6}(2),{ }^{2} E_{6}(2), E_{7}(2)$ and $E_{8}(2)$. Of the latter, completeness of the list of maximal subgroups was claimed only for ${ }^{2} F_{4}(2)^{\prime}$, and a reference is given to [47, although a correction is noted in 24. Subsequently, three cases have been completed and published, namely $F_{4}(2)$ by Norton and Wilson 41, $E_{6}(2)$ by Kleidman and Wilson [25, and $E_{7}(2)$ by Ballantyne, Bates and Rowley [1. The case $E_{8}(2)$ is still not complete, while the proof for ${ }^{2} E_{6}(2)$ has not been published. I have re-calculated the latter case, and can confirm that the list in the Atlas is complete.

Turning now to the sporadic groups and their automorphism groups, just 7 of the 26 cases were unfinished at the time of publication of the Atlas, namely the

Date: 8th April, 2016.

2000 Mathematics Subject Classification. Primary 20D08. 
three Fischer groups, $J_{4}$, the Thompson group, the Monster and the Baby Monster. All except the Monster have now been completed, and lists of maximal subgroups for the simple groups (although not for the automorphism groups) are given in 64. (but note two known errors: the subgroup $O_{8}^{+}(3): S_{4}$ is missing from the list of maximal subgroups of the Baby Monster on page 261, and the subgroup $5^{2}: 2 A_{5}$ of $C o_{1}$ is given wrongly as $5^{2}: 4 A_{5}$ on page 211 ). In this paper we survey the results and main methods, and try to provide full references where the details may be found.

\section{Methods}

2.1. Basic strategy. Suppose $S$ is a sporadic simple group, and $S \leq G \leq \operatorname{Aut}(S)$, and suppose $M \neq S$ is a maximal subgroup of $G$. Then $M \cap S \neq 1$, and if $K$ is a minimal characteristic subgroup of $M \cap S$, then $M=N_{G}(K)$. Since minimal characteristic subgroups of finite groups are direct products of isomorphic simple groups, this leads to a 3 -step process:

(1) determine the characteristically simple subgroups $K$ of $S$, up to conjugacy;

(2) determine the normalizer $N_{G}(K)$ in each case;

(3) decide maximality in $G$ in each case.

Usually Step 1 is by far the most difficult.

There is a fundamental difference between the case when $K$ is an elementary abelian $p$-group (the so-called $p$-local case), and the case when $M \cap S$ has no non-trivial abelian normal subgroup (the so-called non-local case, in which $K$ is necessarily non-abelian). Occasionally $M \cap S$ may have more than one minimal characteristic subgroup, and it may be convenient to dispose of these cases first to avoid duplication of effort. Indeed, even if $M \cap S$ has a unique minimal characteristic subgroup, $M$ itself may not, and such cases may also be treated separately.

2.2. Local subgroups. The techniques of local analysis developed for the classification project for finite simple groups are powerful enough to go quite a long way towards the determination of the maximal $p$-local subgroups. One of the first things to be worked out when a new sporadic group was found was the list of conjugacy classes of elements of prime order, and the corresponding centralizers. This provides a convenient basis for an inductive classification of all $p$-local subgroups.

One starts by finding the conjugacy classes of elements of order $p$ in $N_{S}(\langle x\rangle) /\langle x\rangle$, where $x$ has order $p$. This gives a list of subgroups of order $p^{2}$, and one next determines which of these groups are conjugate, at the same time computing their centralizers, and normalizers. Discarding the cyclic groups of order $p^{2}$, one can then proceed to the next level, classifying the elements of order $p$ in $N_{S}(\langle x, y\rangle) /\langle x, y\rangle$, where $\langle x, y\rangle$ is elementary abelian of order $p^{2}$. And so on. If $G$ has a suitable representation, this process can even be automated, as was done for example by Greg Butler 4 for the 2-local subgroups of the Held group.

Once the Sylow $p$-subgroup gets large, however, this brute-force approach becomes cumbersome, and various refinements are required, especially for $p=2$. For example, if $S$ has a double cover, then the squaring map on an elementary abelian 2 -group in $S$ lifts to a quadratic form, which we may assume is either zero or nonsingular. Indeed, quadratic or symplectic forms can sometimes be constructed in other cases where there are two classes of involutions, even if there is no double cover. For example, in my $\mathrm{PhD}$ thesis I showed that in the Fischer group $F i_{22}$, 
an elementary abelian 2-group which is $2 B$-pure supports an invariant symplectic form, defined to be 1 when the 3 -transposition factors of the two $2 B$-elements fail to commute with each other. In [55] every elementary abelian 2-subgroup of $F i_{24}^{\prime}$ is shown to support an invariant symplectic form.

Similar ideas were used for example by Kleidman and Wilson 27] in $J_{4}$ and by Meierfrankenfeld and Shpectorov [36, 37] in the Monster. More generally, if there is more than one class of elements of order $p$, there is scope for a creative division into the cases that need to be considered.

2.3. Existence of non-local subgroups. In the search for, and classification of, non-local subgroups, the methods will vary depending on whether one is looking for a new simple group or not. I shall assume the latter, and therefore use CFSG when necessary. First consider the problem of proving existence of non-local subgroups. This is surprisingly hard. For example, the embedding of $M_{12}$ in $M_{24}$ was unknown for many years, until proved by Frobenius. When $J_{1}$ was discovered [23], it was conjectured that it contained a subgroup isomorphic to $L_{2}(11)$, but to confirm this required substantial (for those days) computational assistance. Later, Livingstone 34. constructed $J_{1}$ as the automorphism group of a graph on 266 vertices, thereby providing an alternative proof. More recently, the existence of subgroups of the Monster isomorphic to $L_{2}(71)$ 21, $L_{2}(59)$ 20] and $L_{2}(41)$ [3] has been verified only once, again using substantial computational resources.

There are some theoretical methods that can be used, but they have limited applicability. These include the so-called Brauer trick, in which a subgroup is constructed from an amalgam of two subgroups $H$ and $K$ with specified intersection. If there is a representation in which the dimensions of the fixed-point spaces $U, V, W$ of $H, K$ and $H \cap K$ satisfy

$$
\operatorname{dim} U+\operatorname{dim} V>\operatorname{dim} W,
$$

then the group generated by $H$ and $K$ has a fixed point, so is a proper subgroup. More generally, the amalgam method can sometimes be used to identify the subgroup, even when there is no fixed point.

2.4. Non-existence of non-local subgroups. Usually, the hard part of the classification of non-local maximal subgroups is the proof that there are no more than the ones that have been constructed. Non-local subgroups with non-simple socle are relatively easy to classify, as every composition factor of the socle has to lie in the centralizer of an element of prime order $p$, for some $p \geq 5$, which generally reduces the possibilities to a manageable list. So the problem reduces essentially to classifying the non-abelian simple subgroups up to conjugacy.

A reasonable first step is to try to classify them up to isomorphism, using Lagrange's Theorem together with character restriction and knowledge of the $p$-local subgroups to eliminate as many cases as possible. In larger cases, these methods are rarely sufficient, and it will be necessary to carry along a number of 'possible' isomorphism types of simple subgroups for more detailed investigation. For example, Griess [16] was unable, even with the referee's help, to decide if $J_{1}$ is a subgroup of the Monster. This was eventually decided in the negative [53]. The last case in the sporadic groups, namely the question whether $S z(8)$ is contained in the Monster, was decided (in the negative) only very recently [67.

More advanced techniques which we shall discuss in more detail include use of structure constants, restriction of Brauer characters (using [24), computation of 
cohomology, detailed local analysis, and extending normalizers. The first and last of these techniques often reduce to an exhaustive computational search. There is a general tendency to work inductively from small simple groups to large, as exemplified most clearly in Norton's work on the Monster [39], using the normalizer extension method.

\section{Historical SURVEY}

3.1. Livingstone and his students: structure constants. It would appear that the first systematic attempts to classify the maximal subgroups of sporadic simple groups were undertaken by Donald Livingstone and his students in the decade following the discovery of $J_{1}$. While the case of $J_{1}$ itself was straightforward 23. apart from the difficulty of establishing the existence of a subgroup $L_{2}(11)$, the same was not necessarily true for the five previously known sporadic groups, namely the Mathieu groups.

Chang Choi [5, 6] began with $M_{24}$, and completed his thesis in 1968. A particularly troublesome case was the classification of transitive imprimitive subgroups, largely because there was a previously unsuspected maximal subgroup of this type, isomorphic to $L_{2}(7)$. This subgroup was apparently first found by Robert Curtis 9], who went on to provide a new proof for the list of maximal subgroups of $M_{24}$, using his newly-discovered Miracle Octad Generator [8]. This work forms part of his thesis, completed in 1972 under John Conway. Another proof was published by Rudy List [33]. As for the four smaller Mathieu groups, it is not clear that proofs of completeness of their lists of maximal subgroups have even been published. Certainly the literature search conducted while preparing the Atlas did not throw up any such references. Nevertheless, these days such proofs would be regarded as graduate student exercises, and do not present great difficulty.

Two more students of Livingstone, both of whom completed their theses in 1970, were Spyros Magliveras, who determined the maximal subgroups of the HigmanSims group [35, and Larry Finkelstein who did the same for the McLaughlin group and Conway's third group $\mathrm{Co}_{3}$ [13. Finkelstein then collaborated with Arunas Rudvalis to deal with the Janko groups $J_{2}$ [14] and $J_{3}$ [15].

Among the various techniques they used was the method of structure constants. Given three conjugacy classes $C_{1}, C_{2}, C_{3}$ in $G$ there is a well known character formula which counts the number of ways a fixed element $z \in C_{3}$ can be written as the product $z=x y$ of elements $x \in C_{1}$ and $y \in C_{2}$. If the orders of $x, y, z$ are $p, q, r$ respectively, then in certain cases the isomorphism type of $\langle x, y, z\rangle$ is determined:

\begin{tabular}{|ccc|c|}
$p$ & $q$ & $r$ & $\langle x, y, z\rangle$ \\
\hline 2 & 2 & $n$ & $D_{2 n}$ \\
2 & 3 & 3 & $A_{4}$ \\
2 & 3 & 4 & $S_{4}$ \\
2 & 3 & 5 & $A_{5}$ \\
\hline
\end{tabular}

This is particularly useful for classifying subgroups isomorphic to $A_{5}$.

Of note here, however, is the use that was put to calculating structure constants for other triples of integers, especially $(p, q, r)=(2,3,7)$. Since, for example, $L_{2}(7)$ is generated by elements $x$ of order 2 and $y$ of order 3 with $x y$ of order 7, calculation of the structure constants of type $(2,3,7)$ gives an upper bound on the number of subgroups isomorphic to $L_{2}(7)$, and in some cases this bound is actually met. 
3.2. Students of Conway: lattice methods. By the mid-1970s the age of discovery of sporadic simple groups was over, and there were some 14 new groups whose maximal subgroups were waiting to be determined. Up to this point, every case considered had a relatively small permutation representation: the largest was $\mathrm{Co}_{3}$, on 276 points. The next generation of groups needed thousands of points, and demanded new techniques of investigation.

The work of Robert Curtis on maximal subgroups of $M_{24}$ was only part of his thesis, completed in 1972, under John Conway. He also considered in depth the classification of subgroups of the largest Conway group [10, 11. At that time he did not envisage a complete determination of the maximal subgroups, which had to wait another decade. Of particular interest here is his approach to studying subgroups which fix interesting sublattices. This is an extension of Conway's discovery that the Higman-Sims and McLaughlin groups, originally constructed as permutation groups, are also essentially sublattice stabilizers in the Conway group. Curtis discovered several interesting subgroups of $\mathrm{Co}_{1}$ by these methods. He also completely classified the maximal $p$-local subgroups [11. (The case $p=3$ had been done earlier by Mikdashi, a student of Livingstone, but not published except in his $\mathrm{PhD}$ thesis, dated 1971. See also 56 for a correction to 11 for $p=3$.)

Two more of Conway's students worked on subgroups of sporadic groups in the 1970s, and worked out a good deal of the subgroup structure, although apparently without aiming at a full classification of the maximal subgroups. Simon Norton wrote his thesis in 1975 on the group now known as the Harada-Norton group, among other topics. This thesis does not claim to determine the maximal subgroups completely, although he did this later. This work was however not published until a decade later, when I collaborated with him to re-work the determination of the maximal subgroups of the Harada-Norton group [40] and its automorphism group. Gerard Enright wrote his thesis in 1977 on the subgroup structure of the Fischer groups $F i_{22}$ and $F i_{23}$, in which his main result was a classification of the subgroups generated by transpositions [12.

Norton went on to do a huge amount of work on the subgroup structure of the Monster, and by extension, the Baby Monster and the Harada-Norton group, as well as $F i_{24}^{\prime}$ and other groups involved in the Monster. His big idea was to create a table of 'Monstralizer pairs', that is, pairs of subgroups $H$ and $K$ such that $H=C(K)$ and $K=C(H)$. The published version of this table 39] contains all cases where $H$ or $K$ has order divisible by a prime $p$ with $p \geq 11$, but in unpublished work he went further than this.

In 1979 I became a student of Conway, and worked first on the Suzuki group (1782 points) [44, and then the Rudvalis group (4060 points) 47]. The methods were not much different from the methods of Livingstone's students, except perhaps in a greater emphasis on using properties of lattices on which the groups (or, more accurately, their covering groups) act. For example, the 6-fold cover of the Suzuki group acts on the complex Leech lattice, of dimension 12. If one can show by character theory that a subgroup isomorphic to $K$ has a fixed vector in the lattice, then the same is true in the lattice reduced modulo 3 . But there are only two orbits of non-zero vectors in the latter, and the vector stabilizers are already known. Thus the difficult cases to classify are the irreducible subgroups, in which case exhaustive computer searches were carried out. This included construction of the subgroups $A_{7}, L_{2}(25)$ and $L_{3}(3)$. Similarly, in the Rudvalis group, the subgroups $L_{3}(3)$, 
$L_{2}(13)$ and $A_{8}$ were found by computer searches. The subgroups $U_{3}(5)$ and $L_{2}(29)$ had been found much earlier by Kiang-Chuen Young (a student of John McKay in Canada, $\mathrm{PhD}$ thesis 1974), using similar computational methods.

The lattice method really showed its power, however, in the case of $\mathrm{Co}_{2}$, where I used a 23-dimensional sublattice of the Leech lattice [45. The fact that 23 is prime was particularly helpful, as it implied that most proper subgroups were reducible, and very few of those did not have a fixed vector. Indeed, character restriction alone is sufficient to show that every proper non-abelian simple subgroup of $\mathrm{Co}_{2}$ fixes a vector in the 23-dimensional representation. Automorphisms of order 2 swap two fixed vectors and therefore fix their sum, so the only slight difficulty arises for groups which have automorphisms of order 3 , in this case $L_{2}(8), L_{3}(4)$ and $U_{3}(5)$.

It seemed natural then to try to apply the same methods to $\mathrm{Co}_{1}$, acting on the Leech lattice itself [46]. In this case the so-called Suzuki chain of subgroups provides a long list of (quasi-)simple subgroups which act fixed point freely on the Leech lattice, and the degree 24 allows a number of other cases as well, such as $L_{2}(11), L_{2}(23)$ and $L_{2}(25)$. The alternating groups, for example, were classified by first using the structure constants of type $(2,4,5)$ to limit the number of possibilities for $A_{6}$, and then inductively constructing $A_{n}$ from $A_{n-2}$ and $A_{n-3} \times 3$ intersecting in $A_{n-3}$. Only the final three cases, $L_{2}(11), L_{2}(23)$ and $L_{2}(25)$ required computer calculations to complete. At this stage it became clear that the lattice method could also usefully be applied to the cases considered by Magliveras and Finkelstein, namely the Higman-Sims group, the McLaughlin group and Conway's third group. This provided independent proofs for the complete classification of non-local subgroups in these cases [59].

Although my thesis, in common with earlier work, only dealt with the simple groups, and not their automorphism groups, it became clear fairly soon afterwards that it was necessary to deal with the latter case also. In [49. I went through all the relevant cases up to that point, and adjusted the proofs to include the automorphism groups as well, that is, the groups $M_{12}: 2, M_{22}: 2, J_{2}: 2, J_{3}: 2, H S: 2$, $M c L: 2, S u z: 2, H e: 2$ and $H N: 2$.

3.3. Global input. By the early 1980s, then, the number of target groups had been reduced to single figures, and a number of people around the world were attacking them. Greg Butler in Australia (a student of John Cannon) dealt with the Held group, using serious computational methods, for example in the systematic enumeration of 2-local subgroups. Satoshi Yoshiara in Japan had written his thesis (unfortunately in Japanese) on the Suzuki group, and went on to determine the maximal subgroups of the O'Nan group 70. Independently, in Moscow, Ivanov, Tsaranov and Shpectorov [22] did the same, as did I [50]. Our methods were quite different, and it was reassuring to find that we all obtained the same answer. The most difficult part was constructing subgroups isomorphic to $A_{7}, L_{2}(31)$, and $M_{11}$, for which I used computation, whereas the other authors above used detailed geometrical methods.

It was also becoming evident that the problems were getting harder, and from this point on it was rare for the maximal subgroup problem for a single group to be completely solved in a single paper. The Lyons group was considered by Andrew Woldar 69. (a student of Ron Solomon) in his PhD thesis in 1984, in which he also conjectured the existence of a 111-dimensional representation over the field of order 5 . This representation was also conjectured by Meyer and Neutsch, 
and constructed by Richard Parker [38, and was used extensively in my work to complete the determination of the maximal subgroups [51, 52].

3.4. Students of Wilson: hard-core computation. It had long been clear that computational methods were a necessary part of the maximal subgroup project for sporadic groups. Theoretical methods were simply not powerful enough to probe the structure of these unique objects in sufficient detail. In terms of checking the results, it may be felt that these computer searches represent a weak point. Nowadays, however, computer algebra systems such as MAGMA and GAP are sufficiently well developed that it should be possible with relatively little effort, and insignificant amounts of computer time, to reproduce these results robustly. As far as I am aware, this effort has not yet been made, but it is surely time now to do so.

The story of the original hard-core computation really starts with Peter Kleidman, who was a student of Martin Liebeck. When Liebeck moved from Cambridge to London, Peter worked much of the time with me. We first finished the determination of the maximal subgroups of $F i_{22}$, and its automorphism group $F i_{22}$ :2, which I had been unable to complete on my own [48, 26]. Then we attacked $J_{4}$ [27, largely because it had a small representation, of dimension 112 over the field of order 2. We found two new maximal subgroups $U_{3}(3)$ and $M_{22}: 2$. Independently, Wolfgang Lempken [29] classified the maximal $p$-local subgroups of $J_{4}$, but his methods were insufficient to complete the determination of the non-local subgroups. Kleidman and I were then joined by Richard Parker in a project to classify the maximal subgroups of $F i_{23}$.

My first official PhD student was Steve Linton, whose thesis, dated 1990, was on maximal subgroups of the Thompson sporadic simple group $T h$ [30] and the Fischer group $\mathrm{Fi}_{24}^{\prime}$ [31 and its automorphism group. The $p$-local subgroups of $F i_{24}^{\prime}$ had been classified in 55. Linton's work on $T h$ also built on my earlier work [58, which reduced the problem to classifying subgroups isomorphic to $L_{2}(19), A_{6}$, $L_{2}(7), L_{3}(3)$ and $U_{3}(3)$. In particular, he discovered new subgroups $L_{2}(19): 2$ and $L_{3}(3)$. Much of the work was done using computations in the 248-dimensional representation. A major theme of the computations was a systematic enumeration of pairs of elements $(x, y)$ with $x, y$ and $x y$ in specified conjugacy classes.

My next student to work on maximal subgroups of sporadic groups was Petra (Beth) Holmes, whose PhD thesis on 'Computing in the Monster' dates from 2002. But the Monster really deserves a section to itself, as perhaps does the Baby Monster.

3.5. The Baby Monster. Simon Norton had already done a lot of work on the maximal subgroups of the Baby Monster, but most of this has not been published. In [54] I had classified the $p$-local subgroups for $p$ odd, and provided some basic information about non-local subgroups. The 2-local subgroups were classified by Meierfrankenfeld and Shpectorov [36, 37. In 62 about half of the cases of the non-local subgroup problem were dealt with, by theoretical methods. This relied heavily on Norton's work on subgroups of the Monster.

Then in 60] I constructed generators for the Baby Monster as $4370 \times 4370$ matrices over the field of order 2 , which paved the way for a complete determination of the maximal subgroups. Nevertheless, the computations were not straightforward, as at that time a single matrix multiplication took around 20 minutes. The first 
result [61] was the construction of previously unknown maximal subgroups $L_{2}(31)$ and $L_{2}(49)^{\cdot} \cdot 2$. The project was completed in [63, although the promised follow-up paper containing details never appeared.

One technique employed here, which became even more important in the Monster, was to classify subgroups generated by two copies of $A_{5}$ intersecting in $D_{10}$. Since the subgroups $A_{5}$ and $D_{10}$ can both be counted using the structure constants, it is relatively straighforward to enumerate all the cases, and test for isomorphism with any desired group. Other cases were more like the case of $L_{2}(17)$, which can be generated by subgroups 17:8 and $D_{16}$ intersecting in a cyclic group of order 8 . In this case, I found representatives for the three classes of 17:8 in the Baby Monster, and then found the normalizers of the three cyclic groups of order 8 , using standard methods [2] for finding involution centralizers, repeatedly.

3.6. The Monster. The Monster is a special case because of its enormous size, and a large number of papers (at least 15) have been written on various aspects of its subgroup structure. To date 44 conjugacy classes of maximal subgroups are known, and the possibilities for presently unknown maximal subgroups are severely restricted. The $p$-local maximal subgroups for odd $p$ were determined in [57, and for $p=2$ in [36, 37.

The first serious attack on the non-local subgroups was carried out by Simon Norton, whose results are reported in [39], although without detailed proofs. From a classification of subgroups isomorphic to $A_{5}$, obtained in part from the calculation of structure constants, he obtained a complete classification of simple subgroups containing an $A_{5}$ with $5 A$-elements. The method is illustrated by the case $A_{6}$, which can be generated by two copies of $A_{5}$, intersecting in $D_{10}$ (or $S_{4}$ : both methods are useful). Hence the centralizer of any $A_{6}$ is the intersection of two copies of the $A_{5}$-centralizer inside the $D_{10}$-centralizer. The latter is either the Harada-Norton group or an involution centralizer therein, so the calculation reduces to computing double cosets of certain subgroups of $H N$.

This work was extended in 42, which classified simple groups into three categories: those which were definitely in the Monster, those which were definitely not, and those for which we could not decide at that time. These lists are not quite correct, as $L_{2}(41)$ was put in the 'definitely not' category, whereas we now know that $L_{2}(41)$ is in fact a subgroup of the Monster [43]. The case $J_{1}$ was handled in [53.

At that point, a computer construction of the Monster became available, based on the 3-local subgroup $3^{1+12} \cdot 2 \cdot S u z: 2$. This was used by my student Beth Holmes to investigate subgroup structure, but it soon became clear that we needed an involution centralizer, so we built the Monster again [18, this time using $2^{1+24} \cdot \mathrm{Co}_{1}$. The first result of this work was the discovery of a new maximal subgroup $L_{2}(29): 2$ [19, followed closely by $L_{2}(59)$ 20. This was then extended to a systematic study of subgroups generated by two copies of $A_{5}$ with $5 B$-elements [21, which turned up new maximal subgroups $L_{2}(71)$ and $L_{2}(19): 2$. Later, Holmes [17] classified subgroups isomorphic to $S_{4}$, and used this to classify subgroups isomorphic to $U_{3}(3), L_{3}(3), L_{2}(17)$, and $L_{2}(7)$. More recent computations were used to classify subgroups $L_{2}(41)$ [43], $L_{2}(27)$ [65], and $L_{2}(13)$ containing $13 B$-elements [66]. A largely theoretical, but delicate, proof that the Monster does not contain $S z(8)$ is given in 67 . 
At the time of writing, the published results on non-local subgroups of the Monster include complete classifications of maximal subgroups with simple socle, in all cases except when the socle is $L_{2}(8), L_{2}(13), L_{2}(16), U_{3}(4)$ or $U_{3}(8)$. In more recent work, not yet published, I have eliminated the case $U_{3}(8)$ theoretically, and the cases $U_{3}(4)$ and $L_{2}(8)$ computationally. The remaining two cases do not appear to present significantly greater difficulty, and should be completed before long.

\section{Results}

There are very few known errors in the lists of maximal subgroups in the Atlas:

- in $J_{3}$ and $J_{3}: 2$ the shape of the Sylow 3-subgroup is given as $3^{2} \cdot\left(3 \times 3^{2}\right)$, which should be $3^{2} \cdot 3^{1+2}$;

- $L_{2}(17): 2$ is wrongly included as a subgroup of $F i_{23}$;

- in $C_{1}$, the two groups described as $N\left(3 C^{2}\right)$, of shapes $3^{2} \cdot\left[2.3^{6}\right] .2 A_{4}$ and $3^{2} \cdot\left[2^{3} .3^{4}\right] .2 A_{4}$, either do not exist or are not maximal. Also, the subgroup described as $5^{2}: 4 A_{5}$ is actually $5^{2}: 2 A_{5}$.

- in the Monster, the subgroup described as $\left(A_{7} \times\left(A_{5} \times A_{5}\right) .4\right) .2$ should be described as $\left(A_{7} \times\left(A_{5} \times A_{5}\right) \cdot 2^{2}\right) .2$.

Additional information obtained since the publication of the Atlas includes the following:

- the lists for $F i_{22}$ and $F i_{22}: 2$ are complete;

- for $T h$, all listed subgroups exist and are maximal, and the list becomes complete after adding $L_{3}(3)$;

- for $F i_{23}$, the list becomes complete after deleting $L_{2}(17): 2$ and adding $L_{2}(23)$;

- for $J_{4}$, the list becomes complete after adding $M_{22}: 2$ and $U_{3}(3)$;

- for $F i_{24}$ the list is complete, and for $F i_{24}^{\prime}$ becomes complete after adding two classes of $L_{2}(13): 2$.

- for the Baby Monster, all p-local subgroups which are listed without an overgroup are maximal, and the list of maximal subgroups becomes complete on adding the following eight classes of non-local subgroups: $\left(S_{6} \times L_{3}(4): 2\right): 2$, $\left(S_{6} \times S_{6}\right) \cdot 4, L_{2}(49) \cdot 2, L_{2}(31), M_{11}, L_{3}(3), L_{2}(17): 2$, and $L_{2}(11): 2$.

- for the Monster, there is one 7-local maximal subgroup to be added, of shape $7^{2}: S L_{2}(7)$, and five non-local maximal subgroups: $L_{2}(71), L_{2}(59)$, $L_{2}(41), L_{2}(29): 2$, and $L_{2}(19): 2$. Any further maximal subgroup has socle $L_{2}(13)$ or $L_{2}(16)$.

We conclude with tables of results which give an update on the Atlas. In each case, the maximal subgroups are listed in decreasing order of order. To save space, the cases of two conjugacy classes of maximal subgroups of $S$ which are fused in Aut $(S)$ are denoted by the annotation '(two)'. The listing of two maximal subgroups of $M_{12}: 2$ of shape $L_{2}(11): 2$ is not a mistake: there is no automorphism fusing these classes of subgroups, and they are fundamentally different. 


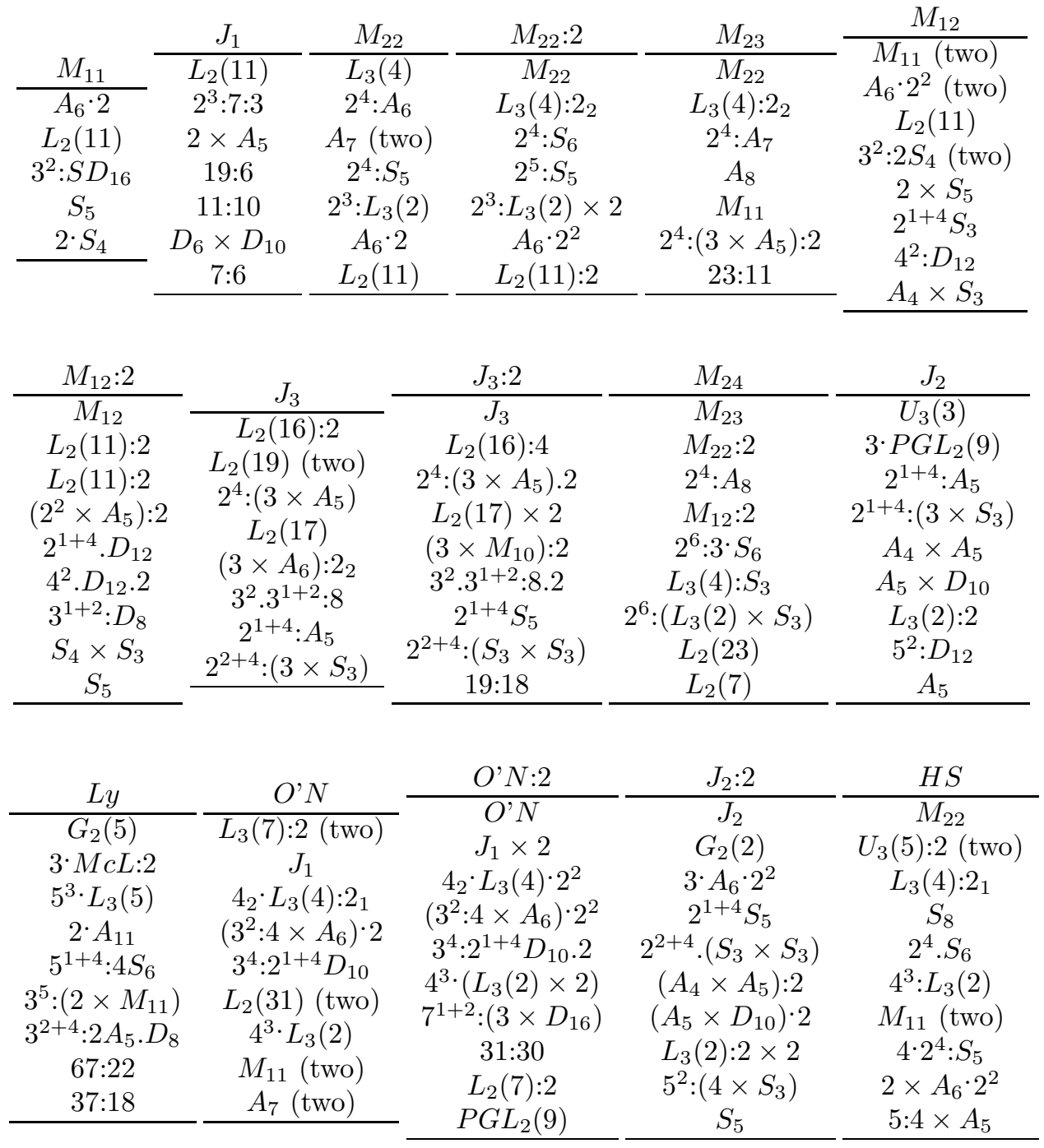

\begin{tabular}{|c|c|c|c|c|}
\hline$H S: 2$ & $M c L$ & $M c L: 2$ & $H e$ & $\frac{\mathrm{Co}_{2}}{\mathrm{C}(2) \cdot 2}$ \\
\hline$H S$ & $U_{4}(3)$ & $M c L$ & $S_{4}(4): 2$ & $U_{6}(2): 2$ \\
\hline$M_{22}: 2$ & $M_{22}($ two $)$ & $U_{4}(3): 2_{3}$ & $2^{2} \cdot L_{3}(4) . S_{3}$ & $2^{10}: M_{22}: 2$ \\
\hline$L_{3}(4): 2^{2}$ & $U_{3}(5)$ & $U_{3}(5): 2$ & $2^{6}: 3 \cdot S_{6}$ (two) & $\begin{array}{c}M c L \\
\text { M }\end{array}$ \\
\hline$S_{8} \times 2$ & $3^{1+4}: 2 S_{5}$ & $3^{1+4}: 4 S_{5}$ & $2^{1+6} \cdot L_{3}(2)$ & $: S_{6}(2)$ \\
\hline $2^{5} \cdot S_{6}$ & $3^{4}: M_{10}$ & $3^{4}:\left(M_{10} \times 2\right)$ & $7^{2}: 2 \cdot L_{2}(7)$ & $\begin{array}{c}H S: 2 \\
\left(2^{1+6} \times 2^{4}\right) A\end{array}$ \\
\hline $4^{3}:\left(L_{3}(2) \times 2\right)$ & $L_{3}(4): 2$ & $\begin{array}{c}L_{3}(4): 2^{2} \\
-S\end{array}$ & $\begin{array}{c}3 \cdot S_{7} \\
7^{1+2} \cdot\left(S_{2} \times 3\right)\end{array}$ & $U_{4}(3) \cdot D_{8}$ \\
\hline$\left(2 \times A_{6} \cdot 2^{2}\right) .2$ & $\begin{array}{c}2 \cdot A_{8} \\
2^{4}: A_{7}(\mathrm{two})\end{array}$ & $\begin{array}{c}2 \cdot S_{8} \\
M_{11} \times 2\end{array}$ & $\begin{array}{c}:\left(S_{3} \times 3\right) \\
S_{4} \times L_{3}(2)\end{array}$ & $2^{4+10}\left(S_{5} \times S_{3}\right)$ \\
\hline $5^{1+2}:\left[2^{5}\right]$ & $M_{11}$ & $5^{1+2}: 3: 8.2$ & $7: 3 \times L_{3}(2)$ & $\begin{array}{l}M_{23} \\
4 . .^{1+4} \mathrm{~S}\end{array}$ \\
\hline $5: 4 \times S_{5}$ & $5^{1+2}: 3: 8$ & $2^{2+4}:\left(S_{3} \times S_{3}\right)$ & $5^{2}: 4 A_{4}$ & $5^{1+2}: 4 S_{4}$ \\
\hline
\end{tabular}




\begin{tabular}{|c|c|c|c|}
\hline & & $F i_{22}: 2$ & $J_{4}$ \\
\hline$\frac{\mathrm{He}: 2}{\mathrm{He}}$ & $\frac{F \imath_{22}}{2 \cdot U_{6}(2)}$ & $F i_{22}$ & $2^{11}: M_{24}$ \\
\hline $\begin{array}{c}H e \\
S_{4}(4): 2\end{array}$ & $O_{7}(3)$ (two) & $2 \cdot U_{6}(2): 2$ & $2^{1+12} \cdot 3 \cdot M_{22}: 2$ \\
\hline $2^{2} \cdot L_{3}(4) \cdot D_{12}$ & $O_{8}^{+}(2): S_{3}$ & $O_{8}^{+}(2): S_{3} \times 2$ & $2^{10}: L_{5}(2)$ \\
\hline $2^{1+6} \cdot L_{3}(2) .2$ & $2^{10}: M_{22}$ & $2^{10}: M_{22}: 2$ & $2^{3+12} \cdot\left(S_{5} \times L_{3}(2)\right)$ \\
\hline $7^{2}: 2 \cdot L_{2}(7) .2$ & $2^{6}: S_{6}(2)$ & $2^{7}: S_{6}(2)$ & $U_{3}(11): 2$ \\
\hline $3 \cdot S_{7} \times 2$ & $\left(2 \times 2^{1+8}: U_{4}(2)\right): 2$ & $\begin{array}{c}\left(2 \times 2^{1+8}: U_{4}(2): 2\right): 2 \\
S_{3} \times U_{4}(3) .2^{2}\end{array}$ & $\begin{array}{c}M_{22}: 2 \\
11^{1+2}:\left(5 \times 2 S_{4}\right)\end{array}$ \\
\hline $\begin{array}{c}\left(S_{5} \times S_{5}\right): 2 \\
2^{4+4}\left(S_{3} \times S_{3}\right) 2\end{array}$ & $\begin{array}{c}S_{3} \times U_{4}(3): 2_{2} \\
\quad 2 F_{4}(2)^{\prime}\end{array}$ & ${ }^{2} F_{4}(2)$ & $L_{2}(32): 5$ \\
\hline $7^{1+2}:\left(S_{3} \times 6\right)$ & $2^{5+8 .} \cdot\left(S_{3} \times A_{6}\right)$ & $2^{5+8}:\left(S_{3} \times S_{6}\right)$ & $L_{2}(23): 2$ \\
\hline$S_{1} \times L_{3}(2) \cdot 2$ & $\begin{array}{r}\cdot\left(3_{3} \times A_{6}\right) \\
3^{1+6} \cdot 2^{3+4} \cdot 3^{2} \cdot 2\end{array}$ & $3^{5}:\left(2 \times U_{4}(2): 2\right)$ & $U_{3}(3)$ \\
\hline $\begin{array}{r}D_{4} \times L_{3} \\
7: 6 \times L\end{array}$ & & $3^{1+6}: 2^{3+4}: 3^{2}: 2.2$ & $29: 28$ \\
\hline $\begin{array}{l}0 \times 1 \\
5^{2}: 45\end{array}$ & $\begin{array}{c}\mathrm{S}_{10}(\mathrm{lW} \\
M_{12}\end{array}$ & $G_{2}(3): 2$ & $43: 14$ \\
\hline & & $M_{12}: 2$ & $37: 12$ \\
\hline
\end{tabular}

\begin{tabular}{|c|c|c|c|}
\hline$H N$ & $H N: 2$ & $\mathrm{Co}_{3}$ & $F i_{23}$ \\
\hline$\frac{H N}{A_{12}}$ & $\frac{H N: 2}{H N}$ & $\overline{M c L: 2}$ & $2 \cdot F i_{22}$ \\
\hline $\begin{array}{r}A_{1} \\
2 \cdot H\end{array}$ & $H \Lambda$ & $H S$ & $O_{8}^{+}(3): S_{3}$ \\
\hline $2^{\circ}$ & $S$ & $U_{4}(3): 2^{2}$ & $2^{2 \cdot} \cdot U_{6}(2) .2$ \\
\hline $2^{1+8}(A$ & 6 & $M_{23}$ & $S_{8}(2)$ \\
\hline$\left(D_{10} \times U_{3}\right.$ & $2^{1+8}\left(A_{5} \times A_{5}\right) .2^{2}$ & $:\left(M_{11} \times 2\right)$ & $S_{3} \times O_{7}(3)$ \\
\hline $5^{1-}$ & $5: 4 \times U_{3}(5): 2$ & $2 \cdot S_{6}(2)$ & $2^{11} \cdot M_{23}$ \\
\hline $2^{6} \cdot U_{4}(2)$ & $5^{1+4} .2^{1+4} \cdot 5.4 .2$ & $\begin{array}{l}3(5): S_{3} \\
3^{1+4}: 4 S_{6}\end{array}$ & $\begin{array}{l}3^{1+0} \cdot 2^{1+0} \cdot 3^{1+2} \cdot 2 S_{4} \\
3^{3} \cdot\left[3^{7}\right] \cdot\left(2 \times L_{3}(3)\right.\end{array}$ \\
\hline $\begin{array}{c}\left(A_{6} \times A_{6}\right) \cdot D_{8} \\
2^{3+2+6}\left(3 \times L_{3}(2)\right)\end{array}$ & $2^{6} \cdot U_{4}(2) .2$ & $2^{4} \cdot A_{8}$ & $S_{12}$ \\
\hline $5^{2+}$ & $2^{3+2+6}\left(S_{3} \times L_{3}(2)\right)$ & $C_{3}(4): D_{12}$ & $\left.{ }^{+8}\right) \cdot\left(3 \times U_{4}(2)\right)$ \\
\hline$M_{12}: 2(\mathrm{two})$ & $5^{2+1+2} 4 S_{5}$ & $2^{2} \cdot\left[2^{7} \cdot 3^{2}\right] \cdot S_{3}$ & $S_{4} \times S_{6}(2)$ \\
\hline $\begin{array}{c}: 2\left(A_{4} \times A_{4}\right) \cdot 4 \\
3^{1+4}: 4 A_{5}\end{array}$ & $\begin{array}{c}3^{4}: 2\left(S_{4} \times S_{4}\right) \cdot 2 \\
3^{1+4}: 4 S_{5}\end{array}$ & $S_{3} \times L_{2}(8): 3$ & $S_{4}(4): 4$ \\
\hline & & & $L_{2}(2$ \\
\hline
\end{tabular}

\begin{tabular}{|c|c|c|}
\hline$B$ (first part) & $B$ (second part) & $B$ (third part) \\
\hline $2^{\cdot 2} E_{6}(2): 2$ & $H N: 2$ & $5^{1+4} \cdot 2^{1+4} \cdot A_{5} \cdot 4$ \\
\hline $2^{1+22} \cdot \mathrm{Co}_{2}$ & $O_{8}^{+}(3): S_{4}$ & $\left(S_{6} \times S_{6}\right) .4$ \\
\hline $\begin{array}{c}F i_{23} \\
2^{9+16} S_{8}(2)\end{array}$ & $\begin{array}{c}3^{1+8} \cdot 2^{1+6} \cdot U_{4}(2) \cdot 2 \\
\left(3^{2} \cdot D \times U^{\prime}(3)-2\right)\end{array}$ & $5^{2}: 4 S_{4} \times S_{5}$ \\
\hline$T h$ & $5: 4 \times H S: 2$ & $L_{2}(31)$ \\
\hline$\left(2^{2} \times F_{4}(2)\right): 2$ & $S_{4} \times{ }^{2} F_{4}(2)$ & $M_{11}$ \\
\hline $2^{2+10+20} \cdot\left(M_{22}: 2 \times S_{3}\right)$ & {$\left[3^{11}\right] .\left(S_{4} \times 2 S_{4}\right)$} & $L_{3}(3)$ \\
\hline$\left[2^{30}\right] \cdot L_{5}(2)$ & $S_{5} \times M_{22}: 2$ & $L_{2}(17): 2$ \\
\hline$S_{3} \times F i_{22}: 2$ & $\left(S_{6} \times L_{3}(4): 2\right): 2$ & $L_{2}(11): 2$ \\
\hline$\left[2^{35}\right] .\left(S_{5} \times L_{3}(2)\right)$ & $5^{3} \cdot L_{3}(5)$ & $47: 23$ \\
\hline
\end{tabular}




\begin{tabular}{|c|c|c|c|}
\hline$R u$ & Suz & Suz:2 & $T h$ \\
\hline$\frac{\kappa u}{{ }^{2} F_{1}(2)}$ & $G_{2}(4)$ & Suz & ${ }^{3} D_{4}(2): 3$ \\
\hline $\begin{array}{l}{ }^{-} F_{4}(2) \\
2^{6} \cdot G_{2}(2)\end{array}$ & $3_{2} \cdot U_{4}(3): 2$ & $G_{2}(4): 2$ & $2^{5 \cdot} L_{5}(2)$ \\
\hline$\left(2^{2} \times S z(8)\right) \cdot 3$ & $U_{5}(2)$ & $3_{2} \cdot U_{4}(3): 2^{2}$ & $2^{1+8} \cdot A_{9}$ \\
\hline $\begin{array}{c}\left(2^{2} \times S z(8)\right): 3 \\
2^{3+8} \cdot L_{3}(2)\end{array}$ & $2^{1+6} \cdot U_{4}(2)$ & $U_{5}(2): 2$ & $U_{3}(8): 6$ \\
\hline $\begin{array}{c}2 \cdot L_{3}(2) \\
U_{3}(5): 2\end{array}$ & $3^{5}: M_{11}$ & $2^{1+6} \cdot U_{4}(2) .2$ & $\left(3 \times G_{2}(3)\right): 2$ \\
\hline $\begin{array}{l}3(3: 2 \\
2^{1+4+6}: S_{5}\end{array}$ & $J_{2}: 2$ & $3^{5}:\left(M_{11} \times 2\right)$ & 3. $\left[3^{8}\right] .2 S_{4}$ \\
\hline$L_{2}(25) \cdot 2^{2}$ & $\begin{array}{c}2^{4+6}: 3 A_{6} \\
\left(A_{4} \times L_{3}(4)\right): 2\end{array}$ & $\begin{array}{c}J_{2}: 2 \times 2 \\
2^{4+6}: 3 S_{6}\end{array}$ & $\begin{array}{c}3^{2} \cdot\left[3^{7}\right] .2 S_{4} \\
3^{5}: 2 S_{6}\end{array}$ \\
\hline $\begin{array}{c}A_{8} \\
\end{array}$ & $2^{2+8}:\left(A_{5} \times S_{3}\right)$ & $\left(A_{4} \times L_{3}(4): 2_{3}\right): 2$ & $5^{1+2}: 4 S_{4}$ \\
\hline $\begin{array}{l}L_{2}(29) \\
5^{2}: 4 S_{5}\end{array}$ & $M_{12}: 2$ & $2^{2+8}:\left(S_{5} \times S_{3}\right)$ & $5^{2}: G L_{2}(5)$ \\
\hline $\begin{array}{c}3 \cdot 4 \Delta_{5} \\
3 \cdot A_{6} \cdot 2^{2}\end{array}$ & $3^{2+4}: 2\left(A_{4} \times 2^{2}\right) .2$ & $M_{12}: 2 \times 2$ & $7^{2}:\left(3 \times 2 S_{4}\right)$ \\
\hline $5^{1+2}:\left[2^{5}\right]$ & $\left(A_{6} \times A_{5}\right) \cdot 2$ & $3^{2+4}: 2\left(S_{4} \times D\right.$ & $L_{2}(19): 2$ \\
\hline$L_{2}(13): 2$ & $\left(3^{2}: 4 \times A_{6}\right) \cdot 2$ & $\left(P G L_{2}(9) \times A_{5}\right): 2$ & $L_{3}(3)$ \\
\hline$A_{6} \cdot 2^{2}$ & $L_{3}(3): 2$ (two) & $\left(3^{2}: 8 \times A_{6}\right) \cdot 2$ & $M_{10}$ \\
\hline $5: 4 \times A_{5}$ & $\begin{array}{l}L_{2}(25 \\
A_{-}\end{array}$ & $L_{2}(25): 2$ & $\begin{array}{c}31: 15 \\
S_{5}\end{array}$ \\
\hline
\end{tabular}

\begin{tabular}{|c|c|c|}
\hline $\mathrm{Co}_{1}$ & $F i_{24}^{\prime}$ & $F i_{24}$ \\
\hline $\begin{array}{c}\mathrm{Co}_{2} \\
3 \cdot \mathrm{Suz}_{2}\end{array}$ & $\begin{array}{c}F i_{23} \\
2 \cdot F i_{22} \cdot 2\end{array}$ & $\begin{array}{l}F i_{24} \\
F i_{24}^{\prime}\end{array}$ \\
\hline $\begin{array}{l}3 \cdot S u z: 2 \\
2^{11}: M_{24}\end{array}$ & $\begin{array}{c}2 \cdot F i_{22}: 2 \\
\left(3 \times O_{8}^{+}(3): 3\right): 2\end{array}$ & $F i_{23} \times 2$ \\
\hline $\mathrm{Co}_{3}$ & $O_{10}^{-}(2)$ & $S_{3} \times O_{\infty}^{+}$ \\
\hline $2^{1+8} \cdot O_{8}^{+}(2)$ & $3^{7 \cdot} \cdot \mathrm{O}_{7}(3)$ & $\begin{array}{l}\times U_{8}(3): \\
O_{10}^{-}(2): 2\end{array}$ \\
\hline $\begin{array}{c}U_{6}(2): S_{3} \\
\left(A+G_{9}(4)\right) \cdot 2\end{array}$ & $\begin{array}{c}3^{1+10}: U_{5}(2): 2 \\
2^{11} \cdot M\end{array}$ & $3^{7} \cdot O_{7}(3): 2$ \\
\hline $\begin{array}{c}\left(A_{4} \times G_{2}(4)\right): 2 \\
2^{2+12}:\left(A_{8} \times S_{3}\right)\end{array}$ & $2^{2} \cdot U_{6}(2): S_{3}$ & $3^{1+10}:\left(U_{5}(2): 2 \times 2\right)$ \\
\hline $2^{4+12} \cdot\left(S_{3} \times 3 S_{6}\right)$ & $2^{1+12} \cdot 3 \cdot U_{4}(3) \cdot 2_{2}$ & $\left(2 \times 2^{2 \cdot} \cdot U_{6}(2)\right): S_{3}$ \\
\hline $\begin{array}{c}3^{2} \cdot U_{4}(3) \cdot D_{8} \\
3^{6}: 2 M_{12}\end{array}$ & $\begin{array}{c}3^{2+4+8} \cdot\left(A_{5} \times 2 A_{4}\right) \cdot 2 \\
3^{3} \cdot\left[3^{10} \cdot G L_{3}(3)\right.\end{array}$ & $\begin{array}{c}\left(2 \times Z^{2}(2)\right): S_{3} \\
2^{1+12 \cdot} \cdot 3 \cdot U_{4}(3) \cdot 2^{2} \\
2^{2+4+8} \cdot(\end{array}$ \\
\hline $\begin{array}{c}\left(A_{5} \times J_{2}\right): 2 \\
3^{1+4}: 2 \cdot U_{4}(2): 2\end{array}$ & $\begin{array}{c}\left(A_{4} \times O_{8}^{+}(2): 3\right): 2 \\
H e: 2(\text { two })\end{array}$ & $3^{3} \cdot\left[3^{10}\right] \cdot\left(L_{3}(3) \times 2^{2}\right)$ \\
\hline $\begin{array}{r}\left(A_{6} \times U_{3}(3)\right): 2 \\
\left.3^{3+4.2} S \times S\right)\end{array}$ & $2^{3+12} \cdot\left(L_{3}(2) \times A_{6}\right)$ & $\begin{array}{c}S_{4} \times O_{8}^{+}(2): S_{3} \\
2^{3+12} \cdot\left(L_{3}(2) \times S_{6}\right)\end{array}$ \\
\hline$A_{9} \times S_{3}$ & $\begin{array}{l}2^{0+8} \cdot\left(S_{3} \times A_{8}\right) \\
\left(3^{2}: 2 \times G_{2}(3)\right) \cdot 2\end{array}$ & $2^{7+8} \cdot\left(S_{3} \times A_{8}\right)$ \\
\hline$\left(A_{7} \times L_{2}(7)\right): 2$ & $\left(A_{5} \times A_{9}\right): 2$ & $\begin{array}{c}\left(S_{3} \times S_{3} \times G_{2}(3)\right): 2 \\
S_{-} \times S_{0}\end{array}$ \\
\hline$\left.)_{10} \times\left(A_{5} \times A_{5}\right) .2\right) .2$ & $A_{6} \times L_{2}(8): 3$ & $\begin{array}{c}\text { S5 } \times S_{9} \\
S_{6} \times L_{2}(8): 3\end{array}$ \\
\hline $\begin{array}{c}5^{1+2}: G L_{2}(5) \\
5^{3 \cdot}\left(4 \times A_{-}\right) 2\end{array}$ & $\begin{array}{c}7: 6 \times A_{7} \\
\text { (3) } \cdot 2\end{array}$ & $7: 6 \times S_{7}$ \\
\hline $\begin{array}{l}5:(4 \times 1 \\
7^{2}:(3 \times 2\end{array}$ & $\begin{array}{l}U_{3}(3): 2 \text { (two) } \\
L_{2}(13): 2 \text { (two) }\end{array}$ & $7^{1+2}:\left(6 \times S_{3}\right) .2$ \\
\hline $5^{2}: 2$ & $29: 14$ & 29: \\
\hline
\end{tabular}




\begin{tabular}{|c|c|c|}
\hline $\mathbb{M}$ (first part) & $\mathbb{M}$ (second part) & $\mathbb{M}$ (third part) \\
\hline $2 \cdot B$ & $5^{1+6}: 2 \cdot J_{2}: 4$ & $\left(S_{5} \times S_{5} \times S_{5}\right): S_{3}$ \\
\hline $2^{1+24} \cdot C_{1}$ & $(7: 3 \times H e): 2$ & $\left(L_{2}(11) \times L_{2}(11): 4\right.$ \\
\hline $3 \cdot F i_{24}$ & $\left(A_{5} \times A_{12}\right): 2$ & $13^{2}: 2 L_{2}(13) .4$ \\
\hline $2^{2.2} E_{6}(2): S_{3}$ & $5^{3+3 \cdot} \cdot\left(2 \times L_{3}(5)\right)$ & $\left(7^{2}:\left(3 \times 2 A_{4}\right) \times L_{2}(7)\right) .2$ \\
\hline $2^{10+16} \cdot O_{10}^{+}(2)$ & $\left(A_{6} \times A_{6} \times A_{6}\right) \cdot\left(2 \times S_{4}\right)$ & $\left(13: 6 \times L_{3}(3)\right) \cdot 2$ \\
\hline $2^{2+11+22} \cdot\left(M_{24} \times S_{3}\right)$ & $\left(A_{5} \times U_{3}(8): 3_{1}\right): 2$ & $13^{1+2}:\left(3 \times 4 S_{4}\right)$ \\
\hline $3^{1+12 \cdot} \cdot 2 \cdot S u z: 2$ & $5^{2+2+4}:\left(S_{3} \times G L_{2}(5)\right)$ & $L_{2}(71)$ \\
\hline $2^{5+10+20} \cdot\left(S_{3} \times L_{5}(2)\right)$ & $\left(L_{3}(2) \times S_{4}(4): 2\right) \cdot 2$ & $L_{2}(59)$ \\
\hline$S_{3} \times T h$ & $7^{1+4}:\left(3 \times 2 S_{7}\right)$ & $11^{2}:\left(5 \times 2 A_{5}\right)$ \\
\hline $2^{3+6+12+18} \cdot\left(L_{3}(2) \times 3 S_{6}\right)$ & $\left(5^{2}:\left[2^{4}\right] \times U_{3}(5)\right) \cdot S_{3}$ & $L_{2}(41)$ \\
\hline $3^{8 \cdot} \mathrm{O}_{8}^{-}(3) \cdot 2_{3}$ & $\left(L_{2}(11) \times M_{12}\right): 2$ & $L_{2}(29): 2$ \\
\hline$\left(D_{10} \times H N\right) \cdot 2$ & $\left(A_{7} \times\left(A_{5} \times A_{5}\right): 2^{2}\right): 2$ & $7^{2}: S L_{2}(7)$ \\
\hline$\left(3^{2}: 2 \times O_{8}^{+}(3)\right) \cdot S_{4}$ & $5^{4}:\left(3 \times 2 \cdot L_{2}(25)\right): 2_{2}$ & $L_{2}(19): 2$ \\
\hline $3^{2+5+10} \cdot\left(M_{11} \times 2 S_{4}\right)$ & $7^{2+1+2}: G L_{2}(7)$ & $41: 40$ \\
\hline $3^{3+2+6+6}:\left(L_{3}(3) \times S D_{16}\right)$ & $M_{11} \times A_{6} \cdot 2^{2}$ & others? \\
\hline
\end{tabular}

[1] J. Ballantyne, C. Bates and P. J. Rowley, The maximal subgroups of $E_{7}(2), L M S$ J. Comput. Math. 18 (2015), 323-371.

[2] J. N. Bray, An improved method for generating the centralizer of an involution, Arch. Math. (Basel) 74 (2000), 241-245.

[3] J. N. Bray, D. F. Holt and C. Roney-Dougal, The maximal subgroups of the low-dimensional finite classical groups, LMS Lecture Notes Ser. 407, Cambridge UP, 2013.

[4] Gregory Butler, The maximal subgroups of the sporadic simple group of Held, J. Algebra 69 (1981), 67-81.

[5] Chang Choi, On subgroups of $M_{24}$. I. Stabilizers of subsets, Trans. Amer. Math. Soc. 167 (1972), 1-27.

[6] Chang Choi, On subgroups of $M_{24}$. II. The maximal subgroups of $M_{24}$, Trans. Amer. Math. Soc. 167 (1972), 29-47.

[7] J. H. Conway, R. T. Curtis, S. P. Norton, R. A. Parker and R. A. Wilson, An Atlas of Finite Groups, Oxford University Press, 1985.

[8] R. T. Curtis, A new combinatorial approach to $M_{24}$, Math. Proc. Cambridge Philos. Soc. 79 (1976), 25-42.

[9] R. T. Curtis, The maximal subgroups of $M_{24}$, Math. Proc. Cambridge Philos. Soc. 81 (1977), $185-192$.

[10] R. T. Curtis, On subgroups of ·0. I. Lattice stabilizers, J. Algebra 27 (1973), 549-573.

[11] R. T. Curtis, On subgroups of ·. II. Local structure, J. Algebra 63 (1980), 413-434.

[12] G. M. Enright, Subgroups generated by transpositions in $F_{22}$ and $F_{23}$, Comm. Algebra 6 (1978), 823-837.

[13] L. Finkelstein, The maximal subgroups of Conway's group $C_{3}$ and McLaughlin's group, $J$. Algebra 25 (1973),58-89.

[14] L. Finkelstein and A. Rudvalis, The maximal subgroups of the Hall-Janko-Wales group, $J$. Algebra 24 (1973), 486-493.

[15] L. Finkelstein and A. Rudvalis, The maximal subgroups of Janko's simple group of order 50, 232, 960, J. Algebra 30 (1974), 122-143.

[16] R. L. Griess, The friendly giant, Invent. Math. 69 (1982), 1-102

[17] P. E. Holmes, A classification of subgroups of the Monster isomorphic to $S_{4}$ and an application, J. Algebra 319 (2008), 3089-3099.

[18] P. E. Holmes and R. A. Wilson, A new computer construction of the Monster using 2-local subgroups, J. London Math. Soc. 67 (2003), 349-364.

[19] P. E. Holmes and R. A. Wilson, A new maximal subgroup of the Monster, J. Algebra 251 (2002), 435-447. 
[20] P. E. Holmes and R. A. Wilson, $P S L_{2}(59)$ is a subgroup of the Monster, J. London Math. Soc. 69 (2004), 141-152.

[21] P. E. Holmes and R. A. Wilson, On subgroups of the Monster containing $A_{5}$ 's, J. Algebra 319 (2008), 2653-2667.

[22] A. A. Ivanov, S. V. Tsaranov and S. V. Shpektorov, Maximal subgroups of the O'Nan-Sims sporadic simple group and its automorphism group, (in Russian) Dokl. Akad. Nauk SSSR 291 (1986), 777-780. English translation: Soviet Math. Dokl. 34 (1987), 534-537.

[23] Z. Janko, A new finite simple group with abelian Sylow 2-subgroups, and its characterization, J. Algebra 3 (1966), 147-186.

[24] C. Jansen, K. Lux, R. A. Parker and R. A. Wilson, An Atlas of Brauer Characters, Oxford UP, 1995.

[25] P. B. Kleidman and R. A. Wilson, The maximal subgroups of $E_{6}(2)$ and Aut $\left(E_{6}(2)\right)$, Proc. London Math. Soc 60 (1990), 266-294.

[26] P. B. Kleidman and R. A. Wilson, The maximal subgroups of $\mathrm{Fi}_{22}$, Math. Proc. Cambridge Philos. Soc. 102 (1987), 17-23. Corrigendum, ibid. 103 (1988), 383.

[27] P. B. Kleidman and R. A. Wilson, The maximal subgroups of $J_{4}$, Proc. London Math. Soc. 56 (1988), 484-510.

[28] P. B. Kleidman, R. A. Parker and R. A. Wilson, The maximal subgroups of the Fischer group Fi23, J. London Math. Soc. 39 (1989), 89-101.

[29] W. Lempken, On local and maximal subgroups of Janko's simple group $J_{4}$, Rend. Accad. Naz. Sci. XL Mem. Mat. 13 (1989), 47-103.

[30] S. A. Linton, The maximal subgroups of the Thompson group, J. London Math. Soc. 39 (1989), 79-88. Corrections, ibid. 43 (1991), 253-254.

[31] S. A. Linton and R. A. Wilson, The maximal subgroups of the Fischer groups $F i_{24}$ and $F i_{24}^{\prime}$, Proc. London Math. Soc. 63 (1991), 113-164.

[32] S. A. Linton, R. A. Parker, P. G. Walsh and R. A. Wilson, A computer construction of the Monster, J. Group Theory 1 (1998), 307-337.

[33] R. J. List, On the maximal subgroups of the Mathieu groups. I. $M_{24}$, Atti Accad. Naz. Lincei Renc. Cl. Sci. Fis. Mat. Natur. 62 (1977), 432-438.

[34] D. Livingstone, On a permutation representation of the Janko group, J. Algebra 6 (1967), $43-55$.

[35] S. Magliveras, The subgroup structure of the Higman-Sims simple group, Bull. Amer. Math. Soc. 77 (1971), 535-539.

[36] U. Meierfrankenfeld and S. V. Shpectorov, Maximal 2-local subgroups of the Monster and Baby Monster, Preprint, Michigan State University, 2002. http://www.mth.msu.edu/ ${ }^{2}$ meier/Preprints/2monster/maxmon.pdf

[37] U. Meierfrankenfeld, The maximal 2-local subgroups of the Monster and Baby Monster, II, Preprint, Michigan State University, 2003.

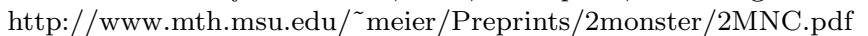

[38] W. Meyer, W. Neutsch and R. A. Parker, The minimal 5-representation of Lyons' sporadic group, Math. Ann. 272 (1985), 29-39.

[39] S. Norton, Anatomy of the Monster: I, in Proceedings of the ATLAS Ten Years On conference (Birmingham 1995), pp. 198-214, Cambridge Univ. Press, 1998.

[40] S. P. Norton and R. A. Wilson, Maximal subgroups of the Harada-Norton group, J. Algebra 103 (1986), 362-376.

[41] S. P. Norton and R. A. Wilson, The maximal subgroups of $F_{4}(2)$ and its automorphism group, Comm. Algebra 17 (1989), 2809-2824.

[42] S. P. Norton and R. A. Wilson, Anatomy of the Monster: II, Proc. London Math. Soc. 84 (2002), 581-598.

[43] S. P. Norton and R. A. Wilson, A correction to the 41-structure of the Monster, a construction of a new maximal subgroup $L_{2}(41)$, and a new Moonshine phenomenon, J. London Math. Soc. 87 (2013), 943-962.

[44] R. A. Wilson, The complex Leech lattice and maximal subgroups of the Suzuki group, J. Algebra 84 (1983), 151-188.

[45] R. A. Wilson, The maximal subgroups of Conway's group ·2, J. Algebra 84 (1983), 107-114.

[46] R. A. Wilson, The maximal subgroups of Conway's group Co1, J. Algebra 85 (1983), 144-165.

[47] R. A. Wilson, The geometry and maximal subgroups of the simple groups of A. Rudvalis and J. Tits, Proc. London Math. Soc. 48 (1984), 533-563. 
[48] R. A. Wilson, On maximal subgroups of the Fischer groups $\mathrm{Fi}_{22}$, Math. Proc. Cambridge Philos. Soc. 95 (1984), 197-222.

[49] R. A. Wilson, Maximal subgroups of automorphism groups of simple groups, J. London Math. Soc. 32 (1985), 460-466.

[50] R. A. Wilson, The maximal subgroups of the O'Nan group, J. Algebra 97 (1985), 467-473.

[51] R. A. Wilson, The subgroup structure of the Lyons group, Math. Proc. Cambridge Philos. Soc. 95 (1984), 403-409.

[52] R. A. Wilson, The maximal subgroups of the Lyons group, Math. Proc. Cambridge Philos. Soc. 97 (1985), 433-436.

[53] R. A. Wilson, Is $J_{1}$ a subgroup of the Monster?, Bull. London Math. Soc. 18 (1986), 349-350.

[54] R. A. Wilson, Some subgroups of the Baby Monster, Invent. Math. 89 (1987), 197-218.

[55] R. A. Wilson, The local subgroups of the Fischer groups, J. London Math. Soc. 36 (1987), $77-94$.

[56] R. A. Wilson, On the 3-local subgroups of Conway's group Co1, J. Algebra 113 (1988), 261-262.

[57] R. A. Wilson, The local subgroups of the Monster, J. Austral. Math. Soc. (A) 44 (1988), $1-16$.

[58] R. A. Wilson, Some subgroups of the Thompson group, J. Austral. Math. Soc. (A) 44 (1988), $17-32$.

[59] R. A. Wilson, Vector stabilizers and subgroups of Leech lattice groups, J. Algebra 127 (1989), $387-408$.

[60] R. A. Wilson, A new construction of the Baby Monster and its applications, Bull. London Math. Soc. 25 (1993), 431-437.

[61] R. A. Wilson, Some new subgroups of the Baby Monster, Bull. London Math. Soc. 25 (1993), 23-28.

[62] R. A. Wilson, More on the maximal subgroups of the Baby Monster, Arch. Math. (Basel) 61 (1993), 497-507.

[63] R. A. Wilson, The maximal subgroups of the Baby Monster, I, J. Algebra 211 (1999), 1-14.

[64] R. A. Wilson, The finite simple groups, Springer GTM 251, 2009.

[65] R. A. Wilson, Classification of subgroups isomorphic to $\mathrm{PSL}_{2}(27)$ in the Monster, LMS J. Comput. Math. 17 (2014), 33-46.

[66] R. A. Wilson, Every $\mathrm{PSL}_{2}(13)$ in the Monster contains $13 A$-elements, LMS J. Comput. Math. 18 (2015), 667-674.

[67] R. A. Wilson, Is Sz(8) a subgroup of the Monster?, Bull. London Math. Soc. 48 (2016), $355-364$.

[68] R. A. Wilson, S. J. Nickerson, J. N. Bray et al., An Atlas of Group Representations, ver. 3, http://brauer.maths.qmul.ac.uk/Atlas/v3/

[69] A. J. Woldar, On the maximal subgroups of Lyons' group, Comm. Algebra 15 (1987), 11951203.

[70] S. Yoshiara, The maximal subgroups of the O'Nan group, J. Fac. Sci. Univ. Tokyo 32 (1985), 105-141.

School of Mathematical Sciences, Queen Mary University of London, London E1 4 NS, U.K.

E-mail address: R.A.Wilson@qmul.ac.uk 